\title{
Does aggressive management of solitary pulmonary nodules pay off?
}

Indeterminate solitary pulmonary nodules (SPNs), measuring up to $3 \mathrm{~cm}$ in diameter, are incidental radiological findings. The ever-growing use of modern imaging has increased their detection. The majority of those nodules are benign; however, the possibility of diagnosing early-stage lung cancer still stands. Guidelines for the management of SPNs have never been validated in prospective comparative studies.

Positron emission tomography (PET) is a useful tool to provide functional information on SPNs. However, overall sensitivity and specificity of PET in detecting malignant SPNs of at least $10 \mathrm{~mm}$ in diameter are about $90 \%$ and false-negative results are reported. The development of video-assisted thoracic surgery has provided minimally invasive diagnosis and treatment of SPNs. In our series, 105 patients underwent surgery based on combined increased ${ }^{18} \mathrm{~F}$-labelled 2-fluoro-2-deoxy-Dglucose (FDG) uptake on PET computed tomography and radiological features (morphology and density) without prior histological confirmation. We detected 26 false negatives (24.8\%) and only nine false positives (8.57\%). Therefore, our minimally invasive surgical approach prevented $25 \%$ of patients with lung cancer from a delayed treatment versus only $9 \%$ undergoing "overtreatment".

In our monocentric cohort, patients with SPNs with large diameter, irregular outline, no calcifications, central location, increased FDG uptake and/or subsolid aspect benefited from a primary surgical resection.

\section{Introduction}

Pulmonary nodules can be incidental findings in scans performed for various indications, including lung cancer screening. Every year, millions of patients are randomly diagnosed with solitary pulmonary nodules (SPNs) on chest imaging or other studies (e.g. computed tomography $(\mathrm{CT})$ urogram, cardiac $\mathrm{CT}$, etc.) and the number is expected to increase in the future with the wider implementation of lung cancer low-dose CT screening worldwide [1-3].

@ERSpublications

There is much debate on the best management of solitary pulmonary nodules. Even if they are mostly benign, they may represent an early-stage lung cancer. Minimally invasive surgical removal is probably the best approach to this insidious disease. http://ow.ly/wMKz30nemjR
Cite as: Elia S, Loprete $S$ De Stefano A, et al. Does aggressive management of solitary pulmonary nodules pay off? Breathe 2019; 15: 15-23.

The need for a systematic approach to these incidental cases overloads the multidisciplinary team, in which radiologists, thoracic surgeons, respiratory physicians, pathologists, palliative care physicians, intensive care specialists, medical and clinical oncologists, clinical nurse specialists and rehabilitation staff are involved. Recently, an increase in SPNs has been identified by highresolution $\mathrm{CT}$ in routine clinics, particularly in lung cancer screening for high-risk populations. Most SPNs are benign and their risk for cancer

\section{CrossMark}

(C) ERS 2019 
increases with size, although it is hard to confidently differentiate small malignant pulmonary nodules from benign ones by $\mathrm{CT}$ features only. Lung cancer probability for nodules measuring $5-6 \mathrm{~mm}$ is $0.9 \%$, while for nodules measuring $10-15 \mathrm{~mm}$ it is $11.1 \%$ [4]. Patients are usually concerned whether an SPN may represent an early-stage lung cancer and, over time, it has become challenging for clinicians to determine whether a nodule is cancerous at its first radiological detection [5]. Therefore, histopathological

Table 1 Aetiology of solitary pulmonary nodules

\begin{tabular}{|c|c|}
\hline Type & Aetiology \\
\hline Infectious & $\begin{array}{l}\text { Tuberculosis } \\
\text { Atypical mycobacteria } \\
\text { Fungal (histoplasmosis, coccidioidomycosis, } \\
\text { cryptococcosis) } \\
\text { Parasitic } \\
\text { Nocardia } \\
\text { Measles } \\
\text { Pneumocystis jirovecii } \\
\text { Round pneumonia } \\
\text { Lung abscess } \\
\text { Septic embolism } \\
\text { Infected bulla }\end{array}$ \\
\hline $\begin{array}{l}\text { Neoplastic } \\
\text { benign }\end{array}$ & $\begin{array}{l}\text { Pulmonary hamartoma } \\
\text { Benign mesenchymal tumours (lipoma, fibroma, } \\
\text { chondroma, haemangioma, leiomyoma) } \\
\text { Neural tumours (schwannoma, neurofibroma, } \\
\text { paraganglioma) } \\
\text { Sclerosing pneumocytoma } \\
\text { Plasma cell granuloma } \\
\text { Endometriosis }\end{array}$ \\
\hline $\begin{array}{l}\text { Neoplastic } \\
\text { malignant }\end{array}$ & $\begin{array}{l}\text { Lung cancer } \\
\text { Neuroendocrine tumours (well differentiated, } \\
\text { moderately differentiated, and poorly differentiated) } \\
\text { Metastasis } \\
\text { Malignant teratoma }\end{array}$ \\
\hline Vascular & $\begin{array}{l}\text { Haematoma } \\
\text { Arteriovenous malformation } \\
\text { Pulmonary artery aneurysm } \\
\text { Pulmonary venous varix } \\
\text { Lung infarct }\end{array}$ \\
\hline Congenital & $\begin{array}{l}\text { Bronchogenic cyst } \\
\text { Lung sequestration }\end{array}$ \\
\hline Inflammatory & $\begin{array}{l}\text { Rheumatoid nodules } \\
\text { Sarcoidosis } \\
\text { Granulomatosis with polyangiitis } \\
\text { Microscopic polyangiitis }\end{array}$ \\
\hline Miscellaneous & $\begin{array}{l}\text { Round atelectasis } \\
\text { Lipoid pneumonia } \\
\text { Amyloidosis } \\
\text { Mucoid impaction } \\
\text { Focal scar }\end{array}$ \\
\hline Lymphatic & $\begin{array}{l}\text { Intrapulmonary lymph node } \\
\text { Lymphoma }\end{array}$ \\
\hline
\end{tabular}

Reproduced and modified from [8] with permission. diagnoses become the vital step in the management of pulmonary nodules, since prompt diagnosis and appropriate management of early lung cancer presenting as an SPN has the highest cure rates, with 5-year survival rate for stage IA reaching up to $80 \%$ [6]. Patients with SPNs are usually asymptomatic and the task for clinicians remains to decide about further management (surveillance or further diagnostics) and review whether surgical resection is indicated, while ensuring the patients are offered balanced information to reach an informed decision. However, the development of video-assisted thoracic surgery (VATS) in the past 15 years currently provides a minimally invasive strategy for both diagnostic and therapeutic excision of SPNs.

\section{Definition criteria for SPNs}

An SPN is usually defined as a rounded or asymmetrical opacity, well or barely demarcated, with a maximum diameter of $3 \mathrm{~cm}$. SPNs are surrounded by normal or emphysematous lung and are not associated with atelectasis, lymphadenopathy or pleural changes, and they do not exhibit features that can be evidently associated with a benign disease. Lung lesions $>3 \mathrm{~cm}$ are classified as masses, while lesions $\leq 3 \mathrm{~mm}$ are generally referred to as micronodules [7, 8].

Possible malignant and benign conditions that may determine the presence of indeterminate lung nodules are listed in table 1

The management of SPNs depends heavily on the probability of malignancy. AL-AMERI et al. [9] published a validation study on four different prediction models of malignancy in pulmonary nodules and demonstrated that even the best among them had the tendency to underestimate the risk in subsequently confirmed malignant nodules, with a mean estimated probability of malignancy of only $34.9 \%$.

Lung cancer is the main cause of cancer death among males; therefore, an SPN must be considered a potential lung cancer until proven otherwise, to avoid delaying treatment of potentially curable early-stage malignancy. However, overemphasising potential malignancy may also lead to unnecessary costs and patient stress. Additionally, the presence of malignancy depends on both patient risk (age, smoking history, underlying tobacco-associated lung disease, exposure to respiratory carcinogens, and other variables) and nodule characteristics. Therefore, the management of SPNs is influenced by different factors, such as the patient's likelihood of lung cancer (pre-test probability), comorbidities, expertise and institutional cultural preferences.

The ideal approach for assessing pulmonary nodules is still vague, since most published guidelines do not clearly state which strategy is accompanied by high-quality evidence among the three that are usually considered: surveillance, non-surgical biopsy and surgical resection. 


\section{Diagnostics for SPNs}

\section{Imaging}

The reported incidence of SPNs on chest radiography ranges from $0.09 \%$ to $7 \%[8,10]$. The detection rates have been improved in recent years by the extensive use of $\mathrm{CT}$ imaging, thanks to its higher spatial resolution, which can visualise nodules of 1-2 $\mathrm{mm}$ in diameter on thin-section scans. Although the clinical relevance of these nodules is quite different from that of larger nodules detected on chest radiographs, the increasing use of $\mathrm{CT}$ scans for various indications will result in almost 1.5 million patients with incidentally detected lung nodules annually, which is going to represent a common clinical problem independent of lung cancer screening [8]. Rates of missed detection of lung cancer on chest radiography range from $20 \%$ to $85 \%$, with smaller, lower attenuation (subsolid) nodules accounting for the majority of them. CT has replaced this technique and is currently the standard imaging procedure to detect and characterise SPNs. All chest CT scans in adults should be reconstructed and archived with contiguous thin sections ( $\leq 1.5 \mathrm{~mm}$, typically $1.0 \mathrm{~mm}$ ) to enable accurate characterisation and measurement of small pulmonary nodules. Routine acquisition and archiving of off-axis (coronal and sagittal) reconstructed series is strongly recommended as well [11].

The attenuation and morphological features of lung nodules on CT are crucial to determine the likelihood of malignancy. Qualitative assessment of nodule CT attenuation measured in the Hounsfield Unit (HU) scale has turned out to be an essential feature of SPNs that influences the probability of malignancy. The presence of calcifications is usually a sign of benign disease, especially when they are symmetrically distributed, even though they have been reported in all histologies of lung cancer [12].

Lung nodule attenuation may be solid or subsolid. Therefore, there are completely nonsolid or ground-glass nodules (GGNs) with a central blurred denseness with normal parenchymal features, or solid nodules with uniform soft-tissue attenuation that replaces lung structure. A subsolid nodule has both characteristics and is rarer but has the highest probability of malignancy, while pure GGNs that persist over sequential examinations are highly suspicious for adenocarcinoma [12]. Current practice suggests surveillance for pure ground-glass lesions detected by thin-slice collimation $>3 \mathrm{~mm}$ and resection or tissue biopsy only if the lesion develops a solid component $>5 \mathrm{~mm}$ [13].

Lung nodule margins may be poorly defined or smooth, lobulated, or spiculated. Spiculated margins are highly associated with malignancy with up to a $90 \%$ positive predictive value [13, 14]. Lobulated margins, even though they are usually associated with benign disease (e.g. hamartoma), carry a higher positive predictive value for well-differentiated neuroendocrine tumours. Nodules with poorly demarcated margins are common to both non-malignant and malignant pathology. Less-differentiating features include the sunburst appearance (corona radiata), upper lobe location, cavitation, vessel sign and pleural $\operatorname{tag}[14,15]$.

The evolutionary features of a subsolid nodule detected during follow-up on CT help in defining its malignant potential. The evolution over time of SPNs is monitored by calculating the volume doubling time measured with nodule volumetry using computer-assisted software programs. Stable size for at least 2 years has long been accepted as confirmation of benignity, while the majority of primary lung cancers demonstrate a doubling time of $<400$ days. Pure GGNs may have very long doubling times; therefore, a minimum 3-year follow-up is required to detect significant change [12]. In subsolid nodules (pure GGNs or partially solid nodules), since attenuation rather than size may anticipate malignant evolution, the assessment of the nodule mass (volumexCT value) has been recently introduced [16].

Dual-energy CT with virtually constructed unenhanced imaging techniques has been recently applied and results in a higher sensitivity (92\%) and accuracy (82\%) in pulmonary nodule characterisation than traditional contrast enhancement (sensitivity $72 \%$, accuracy $71 \%$ ), with the same specificity and lower radiation exposure [17]. Magnetic resonance imaging indications in thoracic assessment are limited by the low signalto-noise ratios within the aerated lung parenchyma, as well as by imaging artefacts related to cardiac and respiratory motion.

Metabolic imaging using ${ }^{18} \mathrm{~F}$-labelled 2-fluoro2-deoxy-D-glucose (FDG) positron emission tomography (PET) is a useful tool to provide functional information on SPNs with a minimum diameter of $8 \mathrm{~mm}$ that cannot be achieved by anatomical cross-sectional imaging alone [18]. The overall sensitivity and specificity of PET in detecting malignant SPNs with a diameter of $\geq 10 \mathrm{~mm}$ is about $90 \%$, yet false-negative results are reported in subsolid nodules such as low-grade neuroendocrine neoplasms (carcinoid tumours) and mucinous adenocarcinomas [19, 20]. When combined with CT (fusion PET-CT) it results in a more accurate characterisation of the nodule and a higher specificity [21]. Moreover, the combination of PET-CT with pre-test clinical risk assessment can better detect the prediction of malignancy, as shown by HERDER et al. [22]. Yı et al. [23] reported that PET-CT was more sensitive (96\% versus $81 \%$ ) and accurate (93\% versus $85 \%$ ) than helical dynamic CT alone. The recent guidelines from the Fleischner Society recommend the use of $\mathrm{PET}-\mathrm{CT}$ in the evaluation of $\geq 8 \mathrm{~mm}$ solid SPNs in both low- and high-risk patients, although the average risk of malignancy in an 8-mm SPN is about 3\% [11]. However, in a study by Nomori et al. [18], $90 \%$ of GGNs, finally 
diagnosed as well-differentiated adenocarcinomas, had a low metabolic uptake. Similarly, inflammatory nodules commonly present as ground-glass or subsolid attenuation, are often metabolically active, and lead to false-positive results [24].

The recently developed four-dimensional PET-CT, or respiratory-gated PET-CT, reduces image misregistration and corrects for respiratory motion, thus improving the assessment of metabolic activity of the nodule. This technique is useful in evaluating nodules located adjacent to the diaphragm, since it reduces the influence of motion on reading the FDG uptake [25].

Quantitative image analysis, also named radiomics, is based on the principle that the biomedical images contain information on tumour phenotype that can be correlated with clinical and molecular data to clarify the presence of malignancy [26]. Such an emerging field continues to grow but requires a multidisciplinary approach to provide reliable data.

\section{Tissue sampling techniques}

Cytological and/or histological diagnosis is the gold standard in definition of SPNs, although less invasive techniques such as liquid biopsy or analysis of exhaled breath for volatile organic compounds are on the increase. Several sampling techniques and methods are currently available, each with strengths and weaknesses. The choice must be made with due consideration of the patient's status, risk of complications, nodule characteristics and institutional expertise.

Contemporary endobronchial techniques are being implemented for tissue sampling, such as radial probe endobronchial ultrasound, virtual navigational bronchoscopy and electromagnetic navigational bronchoscopy, although percutaneous image-guided transthoracic biopsy still remains a crucial minimally invasive technique to obtain diagnostic tissue.

Cytology on fine-needle aspiration biopsy (FNAB) is inadequate for definitive benign diagnosis, while core biopsy provides histological samples with diagnostic accuracy rates for malignancy of between $90 \%$ and $95 \%$. Pneumothorax (8-30\%) and haemorrhage (4-27\%) are the most common complications of transthoracic FNAB, sometimes requiring chest drainage (1-15\%) [27].

\section{Therapeutic modalities for SPNs}

There are several evidence-based recommendations from scientific societies for methodical approaches to managing SPNs [11, 27-29]. Nevertheless, none of these guidelines has been well validated in prospective comparative effectiveness studies. All guidelines take into due consideration multiple factors that may influence decision-making, such as clinical risk, nodule morpho-volumetric features, imaging evolution during surveillance, need for histological diagnosis, risk of complications, comorbidities, and risk of surgical resection.

The recommendations of the Fleischner Society and the American College of Chest Physicians (ACCP) apply to patients with incidentally detected SPNs, yet are based on studies offering relatively limitedquality evidence [11, 30]. The American College of Radiology recommendations for surveillance are even less intensive than for incidentally detected nodules [29]. The British Thoracic Society guidelines recommend no nodule follow-up when the morphology is clearly suggestive of benign disease, for nodules of $<5 \mathrm{~mm}$ in diameter and for perifissural or subpleural nodules of $<10 \mathrm{~mm}$ diameter [28]. Conversely, the ACCP suggests CT surveillance for nodules $<8 \mathrm{~mm}$ in diameter, or for those with a malignant risk of $\leq 5 \%$ [30].

Management of SPNs is a constantly evolving field that matches the improvement of medical culture, technology and expertise. Any strategy concerning SPNs has to consider the likelihood of clinically significant malignancy. Several features contribute to assessment of the risk for malignancy of SPNs: size, attenuation, morphology and location.

The vigorous development of thoracic surgery over the past 20 years, with the routine use of VATS techniques, provides a minimally invasive strategy for diagnostic or therapeutic excision of SPNs. Therefore, minimally invasive surgical resection is currently the treatment of choice for nodules with significant probability of malignancy based on clinical and imaging investigations, or when diagnosis is achieved by means of transthoracic FNAB as recommended by most guidelines. The surgical resection of SPNs without histological diagnosis is still a matter of debate.

Determining nodule localisation has been performed through a variety of techniques, aiming to provide the best aid in video-assisted surgical resection of nodules $<2 \mathrm{~cm}$ in diameter, but without any evidence of superiority for a specific technique. Two studies compared surgical resection with or without pre-operative diagnosis [31, 32], but both were non-randomised and presented quite a few limitations and bias that prevented drawing any significant conclusions. Many studies have addressed the surgical approach (VATS or open lobectomy) and the extent of resection (lobectomy versus segmentectomy or wedge) for nodules diagnosed as malignant, but none has dealt with the specific problem of SPNs. Patients undergoing VATS resection have significantly lower rates of post-operative complications and in-hospital mortality when compared to those undergoing thoracotomy, with no significant difference in 3-year overall survival [33].

As far as the extent of resection is concerned, some studies have demonstrated inferiority of wedge resection compared to segmentectomy, while quite a few non-randomised studies have demonstrated equivalence in the oncological outcomes of lobectomy versus segmentectomy, especially for tumours $<20 \mathrm{~mm}$, 
but better outcomes with lobectomy for larger cancers, as reported in a recent meta-analysis [34]. Lobectomy remains the most widely adopted treatment of nodules with a diagnosis of lung cancer achieved either preoperatively or intra-operatively. When intra-operative frozen section confirms malignancy, resection should be extended for completion lobectomy if previous pulmonary function testing allows an extended procedure. Anatomical video-assisted segmentectomy is the alternative for poorly performing patients, or when location of the nodule precludes a wedge resection and subsequent frozen section [35].

The onset and progressive implementation of awake thoracoscopic surgery has given the go-ahead to multiple surgical procedures in nonintubated patients, thus minimising the patient's discomfort, reducing the complication rate due to general anaesthesia and shortening the length of hospital stay [36, 37]. When the operative risk is too high for the patient to undergo general anaesthesia, awake video-assisted wedge resection represents a valid surgical alternative strategy [38, 39]. Stereotactic ablative radiotherapy or other ablative techniques such as radiofrequency or microwave ablation, for which no superiority compared to surgery has ever been demonstrated, represent in our opinion a less favourable route, to be considered as an alternative only in relevant cases of informed patient choice against surgery or comorbidities precluding surgery [40].

The optimal management approach of nonsolid or partially solid nodules, or GGNs, has been a matter of debate. The management of subsolid pulmonary nodules remains controversial, as there are fewer available historical data to form evidencebased guidelines. Frequently, these nodules are histologically proven to be minimally invasive adenocarcinoma, adenocarcinoma in situ or atypical adenomatous hyperplasia, and this is mainly based on the presence of specific clinical (patient's age) and radiological features (nodule size, bubble-like appearance and pleural retraction or indentation) $[41,42]$. In general, the development of a solid component in a previously pure GGN and the increase in size of the solid component of a subsolid nodule or partially solid nodule over follow-up is predictive of malignancy [43]. Based on surgical outcome and long-term survival reported after surgery for such cases, most guidelines recommend surgical resection when surgical risk, comorbidities and effect on life expectancy have been thoroughly discussed.

\section{Existing guidelines for SPN management}

Clinical management of incidental pulmonary nodules was previously based on a Fleischner Society statement published in 2005 [44]. This was the main reference document until 2013, and since then three new guidelines and one statement have emerged, presenting up-to-date evidence
Table 2 Current guidelines for the management of incidental pulmonary nodules

\begin{tabular}{llc}
\hline Society & Year & Reference \\
\hline American College of Chest Physicians & 2013 & {$[30]$} \\
British Thoracic Society & 2015 & {$[28]$} \\
Fleischner Society & 2017 & {$[11]$} \\
\hline
\end{tabular}

that has changed practice [11, 28, 30, 45]. Table 2 presents the three up-to-date guidelines issued by relevant societies.

Although these guidelines are particularly helpful, they need to be adopted into the national standards and available resources of each country where they are implemented. It seems that these guidelines differ in their recommendations and this could potentially cause confusion and variation in clinical practice among various centres, rather than achieving a single uniform approach. The detailed description of all the aforementioned guidelines is beyond the scope of this article; however, we would like to highlight that these pulmonary nodule guidelines share some common ground, such as nodule size/ consistency and risk assessment. The actual baseline and repeat protocols, as well as the size cut-off, differ among them. The size threshold to exclude patients from further follow-up is $5 \mathrm{~mm}$ for the ACCP and British Thoracic Society guidelines, and $6 \mathrm{~mm}$ for those from the Fleischner Society. This 1-mm difference could potentially have a major impact on resources in big centres with well-established nodule services and it would be very interesting to calculate in such clinical settings how many scans and how much clinician time would be saved. Nodules are further classified into solid and subsolid (pure GGN or partially solid) according to their consistency and this is an important classification for all guidelines to determine further diagnostic work-up or surveillance.

However, further work-up, surveillance and decision-making processes are influenced by factors at the systems level, physician level and patient level [46]. From a systems perspective, local resources are important, as many institutions may lack the infrastructure and resources to ensure multidisciplinary evaluation, or patient databases to ensure they are not lost to follow-up [47]. Physician experience also plays a crucial role in the process, as do patient choice and comorbidities, which need to be taken into account. In the next section, we share some real-life data on SPNs based on our institutional approach in patients with incidental SPNs that underwent surgical resection.

\section{Real-life data on SPNs}

\section{Hypothesis}

Patients with suspicious incidental SPNs and appropriate multidisciplinary evaluation can safely undergo surgical resection without prior histological confirmation. 

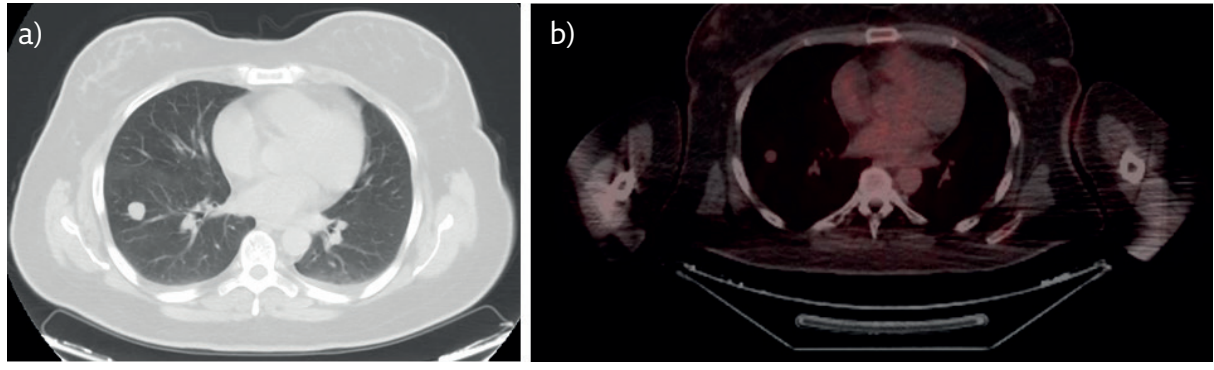

Figure 1 Pulmonary atypical carcinoid of the right lower lobe. a) CT scan showing a polylobate nodule of $15 \mathrm{~mm}$ in diameter. b) The same nodule on PET scan, with SUVmax 1.27 (cut-off 2.5).

\section{Patients and methods}

Our institutional experience concerns a preliminary subset of 105 consecutive patients with SPNs (63 males, 42 females; median (range) age 68 (4486 ) years) who had undergone PET-CT and surgical resection between January 2016 and December 2017. Only SPNs $<3 \mathrm{~cm}$ in diameter within a pattern of normal lung tissue were included. The presence of lymphadenopathy, pleural effusion, radiological features clearly suggesting malignancy or benign disease, and previous cytological diagnosis, were considered as exclusion criteria.

All patients were discussed within our Thoracic Oncology Working Group led by a thoracic surgeon and composed of a representative from each of the departments of respiratory, medical and clinical oncology, radiology, pathology and nuclear medicine.

All patients with a maximum standard uptake value $\left(S U V_{\max }\right)>2.5$ or those with $S U V_{\max }<2.5$ and morphological as well as densitometric features suggesting possible malignancy (spiculated margins, pleural retraction, pleural tag, bronchus sign, attenuation value $>15 \mathrm{HU}$ ) underwent video-assisted wedge resection with frozen section examination. Intra-operative diagnosis of malignancy would indicate completion uniportal video-assisted lobectomy within the same procedure if the patient had been considered fit in the pre-operative work-up.

\section{Results}

No major surgical complications were recorded except for three (2.8\%) prolonged air leaks that required a Heimlich valve in the post-operative period that was removed at 12 days (one patient) or 14 days (two patients), and five $(4.7 \%)$ with exacerbated post-operative pain requiring prolonged administration of intravenous paracetamol. In this cohort, we detected 26 (24.8\%) false negatives, who were essentially the patients who would not be referred for surgery even after a subsequent short-term follow-up (figure 1). Conversely, we found only nine ( $8.57 \%$ ) false-positive cases, who were undoubtedly candidates for surgical resection according to current guidelines for both SUVmax values and morphodensitometric features.

Histology showed primary lung cancer in 76 (72.3\%) patients, metastatic cancer in 10 (9.5\%), six (6.6\%) carcinoids and benign disease in 13 $(12.3 \%)$ cases. Primary adenocarcinoma was the predominant histology $(n=51,67.1 \%)$, followed by squamous $(n=13,17.1 \%)$, neuroendocrine tumours $(n=11,14.4 \%)$ and small cell $(n=1,1.3 \%)$. Interestingly, although not statistically relevant due to the small number of patients enrolled up to now, the subsolid aspect was more associated with malignancy than solid nodules ( $93 \%$ versus $84 \%$ ), even when the latter were characterised by greater SUVmax values (table 3). All patients underwent a minimally invasive uniportal procedure (thoracoscopic video-assisted wedge resection), thanks to the availability of frozen section procedures within the operating theatre, thus avoiding any major post-operative complications. Completion uniportal lobectomy was performed in 65 (79.2\%) patients, while in 17 patients conversion to a muscle-sparing thoracotomy was required (conversion rate 20.7\%), due to severe fibrosis around bronchus or vessels in nine (52.9\%) cases, intra-operative vascular injury and uncontrolled bleeding in four (23.5\%), thick pleural adhesion in two (11.7\%), and fused interlobar

Table 3 Nodule aspect and malignancy

\begin{tabular}{lccccc}
\hline Aspect & Samples & SUV $_{\max }>\mathbf{2 . 5}$ & SUV $_{\max }<\mathbf{2 . 5}$ & Malignant nodules & Benign nodules \\
\hline Solid & 64 & $48(75 \%)$ & $16(25 \%)$ & $54(84 \%)$ & $10(16 \%)$ \\
Subsolid & 41 & $29(71 \%)$ & $12(29 \%)$ & $38(93 \%)$ & $3(7 \%)$
\end{tabular}

Data are presented as $\mathrm{n}$ or $\mathrm{n}(\%)$. Subsolid aspect was more associated with malignancy than solid nodules, even when the latter were characterised by greater SUV max values. 
fissure in two (11.7\%). The 30-day post-operative mortality was zero, while the complication rate was only $2.8 \%$ (prolonged air leak in three cases that required a Heimlich valve, removed in outpatient clinic 12-14 days post-operatively). The mean \pm SD length of post-operative stay was $3.72 \pm 1.33$ days (range 2-8).

\section{Discussion}

SPNs are a diagnostic and therapeutic challenge. Differentiation of malignant from benign lung nodules is a primary concern of lung cancer multidisciplinary teams. Although such lesions are usually benign, the risk of malignancy remains significant. This is true for both lung and metastatic cancer, since surgical resection is the only modality currently offering increased chances of cure and is considered the treatment of choice. In previously reported studies, including the Dutch-Belgian randomised lung cancer screening trial (Nederlands-Leuvens Longkanker Screenings Onderzoek (NELSON)), more than $20 \%$ of participants who underwent low-dose CT screening required a repeat $\mathrm{CT}$, PET imaging, or a biopsy procedure within 12 months after their first screening low-dose CT because of suspicious or intermediate lung nodules. The NELSON trial is the largest European trial, having randomised 15822 subjects. It is powered at $80 \%$ to show a lung cancer mortality reduction of at least $25 \%$, 10 years after randomisation [48]. Although in approximately $25 \%$ of the surgical procedures the nodule was determined to be benign, we believe that VATS has revolutionised the surgical approach by significantly reducing the surgical trauma, the length of hospital stay, post-operative pain and complications, and the duration of recovery of full activity.

The number of patients with SPNs undergoing PET-CT is constantly growing, due to the wider implementation of guidelines and pathways aiming at detecting lung cancer as early as possible to ensure these patients maximise their chance of cure. The management of SPNs is widely dictated by currently available guidelines that relate radiological follow-up and risk stratification

\section{Self-evaluation questions}

1. What is the definition of an SPN?

2. Which is the standard imaging technique to detect an SPN?

3. What are the crucial elements to determine the likelihood of malignancy of an SPN?

4. Which is the most widely adopted treatment of nodules with a diagnosis of lung cancer?

5. What is the NELSON trial?

from low to moderate to high [11]. PET-CT is a noninvasive and highly precise procedure to characterise SPNs with a reported sensitivity of up to $96-97 \%$ and a specificity of $85-88 \%$ [15]. PET-CT plays a crucial role in differentiating nodules $>1 \mathrm{~cm}$ but, for those $<1 \mathrm{~cm}$, in single metastases in some types of malignancies or in benign diseases with increased FDG uptake, it is not conclusive $[18,24]$. For nodules $>8 \mathrm{~mm}$, radiological imaging (CT and $\mathrm{PET}-\mathrm{CT}$ ) is usually accompanied by FNAB and even nodule resection. Therefore, PET-CT complements the clinical and diagnostic evaluation of the patient and a careful radiological analysis of the morphological characteristics of the nodule is required, as demonstrated in our series of patients [49].

\section{Conclusions}

The accurate assessment of risk before additional imaging and volumetric analysis has an important place in lung cancer screening. In the presence of an indeterminate SPN characterised by large diameter, irregular outline, absence of calcifications, central location, increased FDG uptake and subsolid aspect, a surgical resection should be offered. In these cases, where the SUV max is not elevated but morphology is suspicious for malignancy, the possibility of implementing videoassisted minimally invasive surgical excision and frozen section examination in the operating setting helps us in detecting malignancy at an early stage and improves our patients' survival and quality of life.

\section{Affiliations \\ Stefano Elia', Serafina Loprete ${ }^{2}$, Alessandro De Stefano ${ }^{3}$ and Georgia Hardavella 4,5 \\ 1Dept of Surgical Sciences, Thoracic Surgery Unit, Tor Vergata University, Rome, Italy. ${ }^{2}$ Dept of Biomedicine and prevention, Tor Vergata University, Rome, Italy. ${ }^{3}$ Dept of System Medicine, Tor Vergata University, Rome, Italy. ${ }^{4}$ Dept of Respiratory Medicine and Allergy, Medical School, King's College London, London, UK. ${ }^{5} 10$ th Dept of Respiratory Medicine, Athens' Chest Diseases Hospital "Sotiria”, Athens, Greece.}

Conflict of interest

None declared. 


\section{Suggested answers}

1. An SPN is usually defined as a rounded or asymmetrical opacity, well or barely demarcated, with a maximum diameter of $3 \mathrm{~cm}$.

2. CT.

3. The attenuation and morphological features of lung nodules on $\mathrm{CT}$.

4. Lobectomy.

5. Dutch-Belgian randomised lung cancer screening trial.

\section{References}

1. Hall WB, Truitt SG, Scheunemann LP, et al. The prevalence of clinically relevant incidental findings on chest computed tomographic angiograms ordered to diagnose pulmonary embolism. Arch Intern Med 2009; 169: 1961-1965

2. Iribarren C, Hlatky MA, Chandra M, et al. Incidental pulmonary nodules on cardiac computed tomography: prognosis and use. Am J Med 2008; 121: 989-996.

3. Song JH, Beland MD, Mayo-Smith WW. Incidental clinically important extraurinary findings at MDCT urography for hematuria evaluation: prevalence in 1209 consecutive examinations. AJR Am J Roentgenol 2012; 199: 616-622.

4. Horeweg N, Scholten ET, de Jong PA, et al. Detection of lung cancer through low-dose CT screening (NELSON): a prespecified analysis of screening test performance and interval cancers. Lancet Oncol 2014; 15: 1342-1350.

5. Clark ME, Bedford LE, Young B, et al. Lung cancer CT screening: psychological responses in the presence and absence of pulmonary nodules. Lung Cancer 2018; 124: 160-167.

6. Goldstraw P, Chansky K, CrowleyJ, et al. The IASLC Lung Cancer Staging Project: proposals for revision of the TNM stage groupings in the forthcoming (eighth) edition of the TNM classification for lung cancer. J Thorac Oncol 2016; 11: 39-51.

7. Khan $\mathrm{AN}, \mathrm{Al}-J a h d a l i \mathrm{HH}$, Irion $\mathrm{KL}$, et al. Solitary pulmonary nodule: a diagnostic algorithm in the light of current imaging technique. Avicenna J Med 2011; 1: 39-51.

8. Patel VK, Naik SK, Naidich DP, et al. A practical algorithmic approach to the diagnosis and management of solitary pulmonary nodules. Part 1: radiologic characteristics and imaging modalities. Chest 2013; 143: 825-839.

9. Al-Ameri A, Malhotra P, Thygesen $\mathrm{H}$, et al. Risk of malignancy in pulmonary nodules: a validation study of four prediction models. Lung Cancer 2015; 89: 27-30.

10. Gould MK, Tang T, Liu IL, et al. Recent trends in the identification of incidental pulmonary nodules. Am J Respir Crit Care Med 2015; 192: 1208-1214.

11. MacMahon H, Naidich DP, Goo JM, et al. Guidelines for management of incidental pulmonary nodules detected on CT images: from the Fleischner Society 2017. Radiology 2017; 284: 228-243.

12. Ohno $\mathrm{Y}$, Koyama $\mathrm{H}$, Matsumoto $\mathrm{K}$, et al. Differentiation of malignant and benign pulmonary nodules with quantitative first-pass 320-detector row perfusion CT versus FDG PET/CT. Radiology 2011; 258: 599-609.

13. Campo MJ, Lennes IT. Managing patients with screendetected nodules: the nodule clinic. Semin Roentgenol 2017 52: 161-165.

14. Sim YT, Poon FW. Imaging of solitary pulmonary nodule - a clinical review. Quant Imaging Med Surg 2013; 3: 316-326.

15. Mosmann MP, Borba MA, de Macedo FP, et al. Solitary pulmonary nodule and 18F-FDG PET-TC. Part 1: epidemiology, morphological evaluation and cancer probability. Radiol Bras 2016; 49: 35-42

16. de Hoop B, Gietema $H$, van de Vorst $S$, et al. Pulmonary ground-glass nodules: increase in mass as an early indicator of growth. Radiology 2010; 255: 199-206.

17. Sudarski S, Hagelstein C, Weis M, et al. Dual-energy snap-shot perfusion $\mathrm{CT}$ in suspect pulmonary nodules and masses and for lung cancer staging. Eur J Radiol 2015; 84 2393-2400

18. Nomori $\mathrm{H}$, Watanabe $\mathrm{K}$, Ohtsuka $\mathrm{T}$, et al. Evaluation of F-18 fluorodeoxyglucose (FDG) PET scanning for pulmonary nodules less than $3 \mathrm{~cm}$ in diameter, with special reference to the CT images. Lung Cancer 2004; 45: 19-27.

19. Yilmaz F, Tastekin G. Sensitivity of 18 F-FDG PET in evaluation of solitary pulmonary nodules. Int J Clin Exp Med 2015; 8: 45-51.

20. Ruilong Z, Daohai X, Li G, et al. Diagnostic value of 18F-FDG-PET/CT for the evaluation of solitary pulmonary nodules: a systematic review and meta-analysis. Nucl Med Commun 2017; 38: 67-75.

21. Carter BW, Lichtenberger JP3rd, Shroff GS, et al. Role of fluorodeoxyglucose positron emission tomography-computed tomography in the evaluation of suspicious pulmonary nodules. Semin Roentgenol 2017; 52: 166-172.

22. Herder GJ, van Tinteren H, Golding RP, et al. Clinical prediction model to characterize pulmonary nodules: validation and added value of ${ }^{18} \mathrm{~F}$-fluorodeoxyglucose positron emission tomography. Chest 2005; 128: 2490-2496.

23. Yi CA, Lee KS, Kim BT, et al. Tissue characterization of solitary pulmonary nodule: comparative study between helical dynamic CT and integrated PET/CT. J Nucl Med 2006; 47: 443-450.

24. Maiga AW, Deppen SA, Mercaldo SF, et al. Assessment of fluorodeoxyglucose F18-labeled positron emission tomography for diagnosis of high-risk lung nodules. JAMA Surg 2018; 153: 329-334.

25. Callahan J, Kron T, Schneider ME, et al. A prospective investigation into the clinical impact of 4D-PET/CT in the characterisation of solitary pulmonary nodules. Cancer Imaging 2014; 14: 24.

26. Gillies RJ, Kinahan PE, Hricak H. Radiomics: images are more than pictures, they are data. Radiology 2016; 278 : 563-577.

27. Callister ME, Baldwin DR. How should pulmonary nodules be optimally investigated and managed? Lung Cancer 2016; 91: 48-55

28. Callister ME, Baldwin DR, Akram AR, et al. British Thoracic Society guidelines for the investigation and management of pulmonary nodules. Thorax 2015; 70: Suppl. 2, ii1-ii54.

29. Chung K, Jacobs C, Scholten ET, et al. Lung-RADS Category 4X: does it improve prediction of malignancy in subsolid nodules? Radiology 2017; 284: 264-271.

30. Gould MK, Donington J, Lynch WR, et al. Evaluation of individuals with pulmonary nodules: when is it lung cancer? Diagnosis and management of lung cancer, 3rd ed: American College of Chest Physicians evidence-based clinical practice guidelines. Chest 2013; 143: Suppl. 5, e93S-e120S.

31. Heo EY, Lee KW, Jheon $\mathrm{S}$, et al. Surgical resection of highly suspicious pulmonary nodules without a tissue diagnosis. Jpn J Clin Oncol 2011; 41: 1017-1022.

32. Sihoe AD, Hiranandani $\mathrm{R}$, Wong $\mathrm{H}$, et al. Operating on a suspicious lung mass without a preoperative tissue diagnosis: pros and cons. Eur J Cardiothorac Surg 2013; 44: 231-237.

33. Paul S, Isaacs AJ, Treasure T, et al. Long term survival with thoracoscopic versus open lobectomy: propensity matched comparative analysis using SEER-Medicare database. BMJ 2014; 349: g5575

34. Bao F, Ye P, Yang Y, et al. Segmentectomy or lobectomy for early stage lung cancer: a meta-analysis. EurJ Cardiothorac Surg 2014; 46: 1-7

35. Iwata $H$, Shirahashi $K$, Mizuno $Y$, et al. Feasibility of segmental resection in non-small-cell lung cancer with ground-glass opacity. Eur J Cardiothorac Surg 2014; 46: 375-379.

36. Elia S, Guggino G, Mineo D, et al. Awake one stage bilateral thoracoscopic sympathectomy for palmar hyperhidrosis: a safe outpatient procedure. EurJ Cardiothorac Surg 2005; 28: 312-317

37. Pompeo E, Mineo D, Rogliani P, et al. Feasibility and results of awake thoracoscopic resection of solitary pulmonary nodules. Ann Thorac Surg 2004; 78: 1761-1768.

38. Liu J, Cui F, Pompeo E, et al. The impact of nonintubated versus intubated anaesthesia on early outcomes of video-assisted thoracoscopic anatomical resection in nonsmall-cell lung cancer: a propensity score matching analysis. Eur J Cardiothorac Surg 2016; 50: 920-925.

39. Wu CY, Chen JS, Lin YS, et al. Feasibility and safety of nonintubated thoracoscopic lobectomy for geriatric lung cancer patients. Ann Thorac Surg 2013; 95: 405-411.

40. Chang JY, Senan S, Paul MA, et al. Stereotactic ablative radiotherapy versus lobectomy for operable stage I non-smallcell lung cancer: a pooled analysis of two randomised trials. Lancet Oncol 2015; 16: 630-637. 
41. Ichinose J, Kohno T, Fujimori S, et al. Invasiveness and malignant potential of pulmonary lesions presenting as pure ground-glass opacities. Ann Thorac Cardiovasc Surg 2014; 20: 347-352.

42. Fan L, Liu SY, Li QC, et al. Multidetector CT features of pulmonary focal ground-glass opacity: differences between benign and malignant. BrJ Radiol 2012; 85: 897-904.

43. Maeyashiki T, Suzuki K, Hattori A, et al. The size of consolidation on thin-section computed tomography is a better predictor of survival than the maximum tumour dimension in resectable lung cancer. Eur J Cardiothorac Surg 2013; 43: 915-918.

44. MacMahon H, Austin JH, Gamsu G, et al. Guidelines for management of small pulmonary nodules detected on CT scans: a statement from the Fleischner Society. Radiology 2005; 237: 395-400.

45. Bankier AA, MacMahon H, Goo JM, et al. Recommendations for measuring pulmonary nodules at $\mathrm{CT}$ : a statement from the Fleischner Society. Radiology 2017; 285: 584-600.

46. Cabana MD, Rand CS, Powe NR, et al. Why don't physicians follow clinical practice guidelines? A framework for improvement. JAMA 1999; 282: 1458-1465.

47. Simmons J, Gould MK, laccarino J, et al. Systems-level resources for pulmonary nodule evaluation in the United States: a national survey. Am J Respir Crit Care Med 2016; 193: 1063-1065.

48. Walter JE, Heuvelmans MA, de Jong PA, et al. Occurrence and lung cancer probability of new solid nodules at incidence screening with low-dose CT: analysis of data from the randomised, controlled NELSON trial. Lancet Oncol 2016; 17: 907-916.

49. Snoeckx A, Reyntiens P, Desbuquoit D, et al. Evaluation of the solitary pulmonary nodule: size matters, but do not ignore the power of morphology. Insights Imaging 2018; 9: 73-86. 